\title{
A Numerical Method for Accelerating the Convergence of the Power Method
}

\author{
Ekhlass S. Al-Rawi \\ Department of Mathematics \\ College of Computers and Mathematical Sciences \\ University of Mosul
}

Received

25 / 02 / 2008
Accepted

22 / 07 / 2008

\section{الخلاصة}

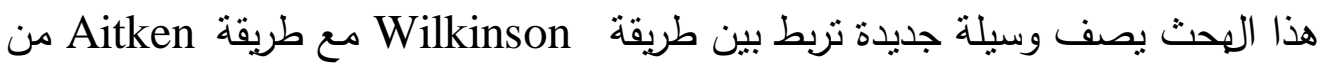

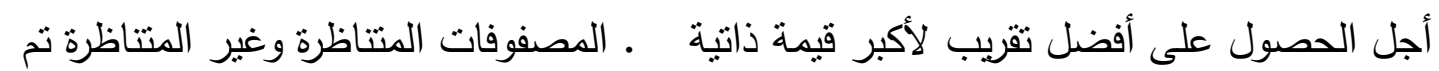

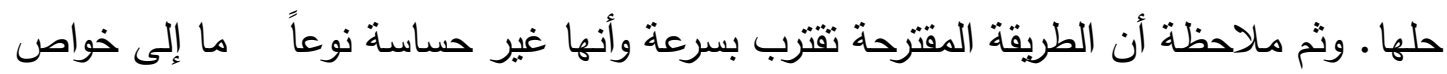

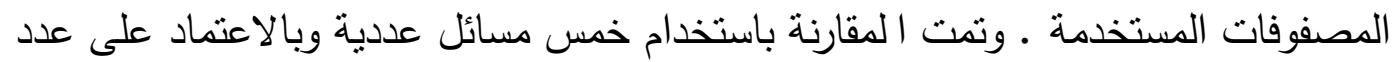

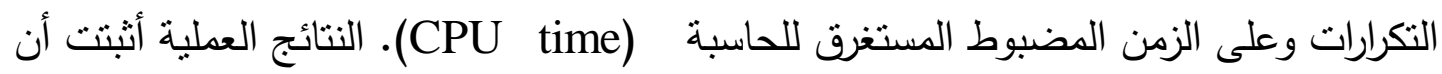

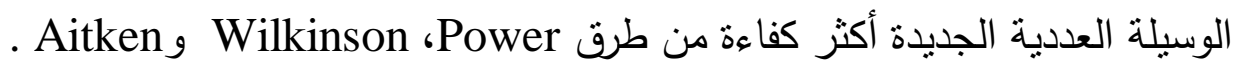

\begin{abstract}
This paper describes a procedure which combines between the Wilkinson and Aitken methods in order to obtain a best approximation of the greatest eigenvalue. Both the symmetric and the nonsymmetric matrices are solved. It shows that our suggested method converges quickly and it is quit insensitive to the properties of the matrices used. A comparison between these approximations for five numerical examples is given, depending on the number of iterations and running computer time. Experimental results indicate that the new numerical procedure is more efficient than Power, Wilkinson and Aitken methods.
\end{abstract}




\section{Introduction}

Eigenvalues arise in many physical problems. Some of the physical situations where eigenvalues are required can be listed: flexural oscillations of a tapered rod, vibration problems, stability problems, buckling of columns, stability of electrical circuits etc. While trying to analyse the solution to these type of problems, we come across eigenvalue problem. These physical problems require for their solution the values of $\lambda$ which satisfy the characteristic polynomial $\mathrm{P}(\lambda)=\operatorname{det}(\mathrm{A}-\lambda \mathrm{I})=0[10]$.

The problem we are considering is this: Given an $n \times n$ real matrix A, find numerical approximations to the eigenvalues and eigenvectors of A. This numerical eigenproblem is difficult to solve in general. In many applications, A may be symmetric, or tridiagonal or have some other special form or property. Consequently, most numerical methods are designed for special matices [3,9]. If $\lambda_{1}$ is an eigenvalue of $A$ that is larger in absolute value than any other eigenvalue, it is called the dominant eigenvalue. An eigenvector $V_{1}$ corresponding to $\lambda_{1}$ is called a dominant eigenvector [6].

Jennings [5] showed from theoretical considerations of Aitkin's process for accelerating the convergence of iterative processes involving matrices and with test examples to overcome the basic unreliability of Aitkin's method for a significant range of applications. Wilkinson [12] described a technique for calculating the eigenvectors of a matrix automatically by means of the Lanczos transformation. This method can be made to give accurate results for both of the Lanczos transformations. Wood et al. [13] described and contrasted the structure and implementation of a new general iterative method for diagonalising large matrices with other more commonly used iterative techniques. The method requires the direct diagonalisation of only a small submatrix, does not require the storage of the large matrix and provides eigensolutions to within a prescribed precision in a rapidly convergent iterative procedure. This technique makes possible efficient solution of a variety of quantum mechanical matrix problems where large basis set expansions are required. Canright et al. [3] introduced a fully distributed Power method for finding the principal eigenvalue of arbitrary matrices that are stored in weighted links of an overlay network. We addressed the problem of synchronization and normalization. Through the example of link analysis we demonstrated both theoretically and experimentally that the proposed method is competitive with the Power method. We have also demonstrated extreme fault tolerance. Al-Bayti and Ahmed [1] produced new technique to improve and calculate the eigenvalues and eigenvectors of the linear system. The procedure is compared with the well-known Power and Aitken methods with promising numerical results. Sun and 
Wei [11] presented some notes of the PageRank algorithm, including its $\mathrm{L}_{1}$ condition number and some observation of the numerical tests of two variant algorithms (standard Power method and Aitken extrapolation) which are based on the extrapolation method.

In this paper, the convergence of the Power method is improved to calculate the greatest eigenvalue of any matrix by using Wilkinson's method and Aitkin's method, and also suggested a new algorithm which combines between the Wilkinson with the Aitken methods in order to obtain the best approximation.

\section{The Power Method}

Assume that the $\mathrm{n} \times \mathrm{n}$ matrix $\mathrm{A}$ has $\mathrm{n}$ distinct eigenvalues $\lambda_{1}$, $\lambda_{2}, \ldots, \lambda_{\mathrm{n}}$ and that they are ordered in decreasing magnitude: that is,

$$
\left|\lambda_{1}\right|>\left|\lambda_{2}\right| \geq\left|\lambda_{3}\right| \geq \ldots \geq\left|\lambda_{n}\right|
$$

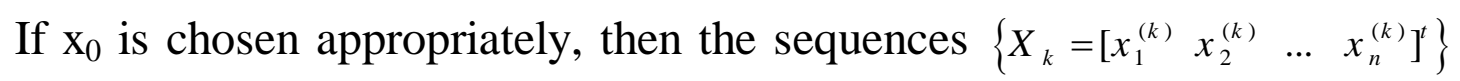
and $\left\{c_{k}\right\}$ generated recursively by

$$
Y_{k}=\mathrm{A} X_{k}
$$

and

$$
X_{k+1}=\frac{1}{c_{k+1}} Y_{k}
$$

where $\quad c_{k+1}=x_{j}^{(k)}$ and $x_{j}^{(k)}=\max _{1 \leq i \leq n}\left\{\left|x_{i}^{(k)}\right|\right\} \quad$ will converge to the dominant eigenvector $V_{1}$ and eigenvalue $\lambda_{1}$, respectively [7]. That is $\lim _{k \rightarrow \infty} X_{k}=V_{1} \quad$ and $\quad \lim _{k \rightarrow \infty} c_{k}=\lambda_{1}$

The rate of convergence of the Power method depends upon how fast the ratios $\left(\lambda_{i} / \lambda_{1}\right)^{k}$ go to zero. Therefore the Power method may converge slowly when the roots are very much closer and hence we would like to accelerate the convergence by some other techniques $[4,11]$.

\section{Algorithm 1 (Power Method): [ 1 ]}

To approximate the dominant eigenvalues and an associated eigenvector of the $\mathrm{nxn}$ matrix $\mathrm{A}$ given a non-zero vector $\mathrm{X}$.

INPUT dimension $\mathrm{n}$, matrix $\mathrm{A}$, vector $\mathrm{X}$, tolerance TOL. Maximum number of iterations $\mathrm{N}$.

OUTPUT approximate eigenpair $(\lambda, X)$ with $\|X\|_{\infty}=1$ or a message that the maximum number of iterations is exceeded

Step 1: Set k=1 
Step 2: Find an integer $\mathrm{p}$ with $1 \leq \mathrm{p} \leq \mathrm{n}$ and $\left|x_{p}\right|=\|X\|_{\infty}$

Step 3: Set $X=\frac{1}{x_{p}} X$

Step 4: If $(k>N)$ goto step 12

Step 5: Set $\mathrm{Y}=\mathrm{AX}$

Step 6: Set $\mu=y_{p}$

Step 7: Find an integer $\mathrm{p}$ with $1 \leq \mathrm{p} \leq \mathrm{n}$ and $\left|y_{p}\right|=\|Y\|_{\infty}$

Step 8: If $\mathrm{y}_{\mathrm{p}}=0$ stop and start with a new vector $\mathrm{X}$.

Step 9: Set $E R R=\left\|X-\left(1 / y_{p}\right) Y\right\|_{\infty}$ and $\mathrm{X}=\left(1 / \mathrm{y}_{\mathrm{p}}\right) \mathrm{Y}$

Step 10: If ERR $<$ TOL then output $(\mu, \mathrm{X})$ (procedure completed successfully) Stop.

Step 11: Else set $\mathrm{k}=\mathrm{k}+1$ goto step 5 .

Step 12: Output (Maximum number of iterations exceeded); (procedure completed unsuccessfully) stop.

\section{The Wilkinson's Method}

Suppose that all the eigenvalues are real but that the Power method is converging slowly because there are two eigenvalues nearly equal in magnitude. By the theory of eigenvalues, we know that A and A-qI have the same eigenvectors. If $\lambda$ is the eigenvalue of $\mathrm{A}$, then $(\lambda-q)$ is the eigenvalue of (A-qI) and the eigenvector in both the cases being same. The optimal choice of $\mathrm{q}$ if we wish to converge to $\lambda_{1}$ is that value which minimizes $\max _{2 \leq i \leq n}\left|\frac{\lambda_{i}-q}{\lambda_{1}-q}\right|$. Hence we have

$$
\mathrm{A} X=\lambda X
$$

and

$$
(\mathrm{A}-q \mathrm{I}) X=(\lambda-q) X
$$

now, we proceed to find the largest eigenvalue of (A-qI) (given in eq.(4)) using the Power method. By a judicious choice of $q$, it may be possible to speed up the convergence markedly. The Power method applied on the new system (A-qI) would give the largest eigenvalue of (A-qI) which is the smallest eigenvalue of $\mathrm{A}$. It is found that, if all the eigenvalues are positive, the optimum value of $\mathrm{q}$ is $\frac{\lambda_{2}+\lambda_{n}}{2}$ where $\lambda_{n}$ is the smallest eigenvalue of A. If we choose $q=\lambda_{n}$, there is also an improved rate of convergence. The iteration technique with $q=\frac{\lambda_{2}+\lambda_{n}}{2}$ will have maximum rate of convergence to $\lambda_{1}-q$ and alternatively with 
$q=\frac{\lambda_{1}+\lambda_{n-1}}{2}$ we have maximum rate of convergence to $\lambda_{n}-q$. Hence the choice of $\mathrm{q}$ depends on the eigenvalues $\lambda_{1}, \lambda_{2}, \ldots, \lambda_{n}$ of the matrix $\mathrm{A}[10,13]$. Algorithm 2 (Wilkinson Method):

INPUT dimension $\mathrm{n}$, matrix $\mathrm{A}$, vector $\mathrm{X}$, tolerance TOL. Maximum number of iterations $\mathrm{N}$.

OUTPUT approximate eigenpair $(\lambda, X)$ with $\|X\|_{\infty}=1$ or a message that the maximum number of iterations is exceeded

Step 1: Find $q=\frac{\lambda_{2}+\lambda_{n}}{2}$

Step 2: Evaluate A-qI

Step 3: Set k=1

Step 4: Find an integer $\mathrm{p}$ with $1 \leq \mathrm{p} \leq \mathrm{n}$ and $\left|x_{p}\right|=\|X\|_{\infty}$

Step 5: Set $X=\frac{1}{x_{p}} X$

Step 6: If $(k>N)$ goto step 14

Step 7: Set $Y=(A-q I) X$

Step 8: Set $\mu=y_{p}$

Step 9: Find an integer $\mathrm{p}$ with $1 \leq \mathrm{p} \leq \mathrm{n}$ and $\quad\left|y_{p}\right|=\mid Y \|_{\infty}$

Step 10: If $\mathrm{y}_{\mathrm{p}}=0$ stop and start with a new vector $\mathrm{X}$.

Step 11: Set $E R R=\left\|X-\left(1 / y_{p}\right) Y\right\|_{\infty}$ and $\mathrm{X}=\left(1 / \mathrm{y}_{\mathrm{p}}\right) \mathrm{Y}$

Step 12: If ERR < TOL then output $(\mu, X)$ (procedure completed successfully) Stop.

Step 13: Else set $\mathrm{k}=\mathrm{k}+1$ goto step 7 .

Step 14: Output (Maximum number of iterations exceeded); (procedure completed unsuccessfully) stop.

\section{The Aitken's Method}

This process uses the form

$$
\dot{\mu}=\mu_{0}-\frac{\left(\mu_{1}-\mu_{0}\right)^{2}}{\mu-2 \mu_{1}+\mu_{0}}
$$

which is approximation to the greatest eigenvalue $\lambda_{1}$, where $\mu_{0}, \mu_{1}$, and $\mu$ are the eigenvalues in the first step, second step and third step respectively, which are obtained from the Power method. This method is 
applicable to any linearly convergence sequence, the rate of convergence of this method for symmetric matrices is $\mathrm{O}\left(\left(\lambda_{2} / \lambda_{1}\right)^{2 m}\right)$ see [3 ].

Algorithm 3 (Aitken Method ): [1]

It is modification of Power algorithm

INPUT dimension $\mathrm{n}$, matrix $\mathrm{A}$, vector $\mathrm{X}$, tolerance TOL. Maximum number of iterations $\mathrm{N}$.

OUTPUT approximate eigenpair $(\lambda, X)$ with $\|X\|_{\infty}=1$ or a message that the maximum number of iterations is exceeded.

Step 1: $\mathrm{k}=1$ set $\mu_{0}=0, \mu_{1}=0$

Step 2: If $(\mathrm{k}>\mathrm{N})$ goto step 10

Step 3: Set $Y=A X$

Step 4: Set $\mu=y_{p} ; \quad \dot{\mu}=\mu_{0}-\frac{\left(\mu_{1}-\mu_{0}\right)^{2}}{\mu-2 \mu_{1}+\mu_{0}}$

Step 5: Find an integer $\mathrm{p}$ with $1 \leq \mathrm{p} \leq \mathrm{n}$ and $\left|y_{p}\right|=\|Y\|_{\infty}$

Step 6: If $\mathrm{y}_{\mathrm{p}}=0$ stop and start with a new vector $\mathrm{X}$.

Step 7: Set ERR $=\left\|X-\left(1 / y_{p}\right) Y\right\|_{\infty}$ and $\mathrm{X}=\left(1 / \mathrm{y}_{\mathrm{p}}\right) \mathrm{Y}$

Step 8: If ERR <TOL then output $(\mu, \mathrm{X})$ (procedure completed successfully) stop.

Step 9: Else set $\mathrm{k}=\mathrm{k}+1$

$$
\begin{aligned}
& \mu_{0}=\mu_{1} \\
& \mu_{1}=\mu
\end{aligned}
$$

Goto step 3

Step 10: Output (Maximum number of iterations exceeded and procedure completed unsuccessfully) stop.

\section{The Suggested Method}

This method combines between the Wilkinson method and Aitken method in order to generate the best approximation. Firstly in this method we compute the matrix (A-qI) where the optimal choice of $q$ is that value which minimizes

$$
\max _{2 \leq i \leq n}\left|\frac{\lambda_{i}-q}{\lambda_{1}-q}\right|
$$

that is the optimum value of $\mathrm{q}$ is $\frac{\lambda_{2}+\lambda_{n}}{2}$, where $\lambda_{n}$ is the smallest eigenvalue of $\mathrm{A}$, and then we use the Power method to find the dominant 
eigenvalue. Secondly we apply the Aitken formula to results from the first step. This iteration technique with $q=\frac{\lambda_{2}+\lambda_{n}}{2}$ will have maximum rate of convergence to $\lambda_{1}-q$, and if the matrix $\mathrm{A}$ has rank $2 \times 2$ then we choose $q=\lambda_{n}=\lambda_{2}$, there is also an improved rate of convergence. We will show that the suggested method converges fastly.

Theorem(1): Let a real sequence $\mu_{n}(n=1,2, \ldots)$ converge to $\lambda$ so that $d_{n} \neq 0$ where $d_{n}=\mu_{n}-\lambda$ for large enough $\mathrm{n}$ and let

$$
\frac{d_{n+1}}{d_{n}} \rightarrow \alpha(n \rightarrow+\infty),|\alpha|<1
$$

If we define $\dot{\mu}_{n}$ by

$$
\dot{\mu}_{n}=\mu_{n}-\frac{\left(\mu_{n+1}-\mu_{n}\right)^{2}}{\mu_{n+2}-2 \mu_{n+1}+\mu_{n}}
$$

then

$$
\frac{\dot{\mu}_{n}-\lambda}{\mu_{n}-\lambda} \rightarrow 0, \quad(n \rightarrow \infty)
$$

Therefore $\dot{\mu}_{n}$ converges to $\lambda$ faster than $\mu_{n}$.

Proof: See [ 8 ].

Theorem(2): Let $\mu_{n}(n=1,2, \ldots)$ satisfy the conditions of Theorem (1) then

$$
\frac{\mu_{n}^{*}-\lambda}{\mu_{n}-\lambda} \rightarrow 0, \quad(n \rightarrow+\infty)
$$

Therefore $\mu_{n}^{*}$ converges to $\lambda$ faster than $\mu_{n}$.

Proof: Using Theorem (1), we have

$d_{n}=\mu_{n}-\lambda-q+q=\left(\mu_{n}-q\right)-(\lambda-q)$, for large enough $\mathrm{n}$ and

$$
\frac{d_{n+1}}{d_{n}} \rightarrow \hat{A}(n \rightarrow+\infty),|\hat{A}|<1
$$

Taking the ratio of the eigenvalues of the proposed algorithm, we have:

$$
\begin{aligned}
\frac{\mu_{n}^{*}-\lambda}{\mu_{n}-\lambda} & =1-\frac{\left(\Delta \mu_{n}\right)^{2}}{\left(\mu_{n}-\lambda\right) \Delta^{2} \mu_{n}} \\
& =1-\frac{\left(\sum_{t=0}^{1}(-1)^{t}\left(\begin{array}{l}
1 \\
t
\end{array}\right) \hat{A}^{1-t}\right)^{2}}{\sum_{t=0}^{2}(-1)^{t}\left(\begin{array}{l}
2 \\
t
\end{array}\right) \hat{A}^{2-t}} \\
& =1-\frac{(\hat{A}-1)^{2}}{(\hat{A}-1)^{2}}=0, \text { where } n \rightarrow+\infty
\end{aligned}
$$


This completes the proof.

\section{Algorithm 4 (New):}

INPUT dimension $\mathrm{n}$, matrix $\mathrm{A}$, vector $\mathrm{X}$, tolerance TOL. Maximum number of iterations $\mathrm{N}$.

OUTPUT approximate eigenpair $(\lambda, X)$ with $\|X\|_{\infty}=1$ or a message that the maximum number of iterations is exceeded.

Step 1: Find $q=\frac{\lambda_{2}+\lambda_{n}}{2}$

Step 2: Evaluate A-qI

Step 3: Set $\mathrm{k}=1$ and $\mu_{0}=0, \mu_{1}=0$

Step 4: If $(k>N)$ goto step 12

Step 5: Set $\mathrm{Y}=(\mathrm{A}-\mathrm{qI}) \mathrm{X}$

Step 6: Set $\mu=y_{p}$

Step 7: Find an integer $\mathrm{p}$ with $1 \leq \mathrm{p} \leq \mathrm{n}, \quad\left|y_{p}\right|=\|Y\|_{\infty}$

and compute $\mu^{*}=\mu_{0}-\frac{\left(\mu_{1}-\mu_{0}\right)^{2}}{\mu-2 \mu_{1}+\mu_{0}}$

Step 8: If $\mathrm{y}_{\mathrm{p}}=0$ stop and start with a new vector $\mathrm{X}$.

Step 9: Set ERR $=\left\|X-\left(1 / y_{p}\right) Y\right\|_{\infty}$ and $\mathrm{X}=\left(1 / \mathrm{y}_{\mathrm{p}}\right) \mathrm{Y}$

Step 10: If ERR <TOL then output $(\mu, \mathrm{X})$ stop.

Step 11: Else set $\mathrm{k}=\mathrm{k}+1$

$$
\begin{aligned}
& \mu_{0}=\mu_{1} \\
& \mu_{1}=\mu
\end{aligned}
$$

Goto step 5

Step 12: Output (Maximum number of iterations exceeded) stop.

\section{Numerical Results}

In this section we present five numerical examples to test the effectiveness of these methods and compare the suggested algorithm with the usual Power, Wilkinson and Aitken methods, where identical Matlab7 programs are used and R.T. is running time (CPU time).

Example (1): [6 ] consider the matrix

$$
A=\left[\begin{array}{ll}
1 & 6 \\
9 & 2
\end{array}\right]
$$

The exact eigenvalues of $\mathrm{A}$ are $\lambda_{1}=8.8655, \lambda_{2}=-5.8655$ 
we compute $q=\lambda_{2}=-5.8655$

The numerical values of this solution are presented in table (1).

Table (1): Results of the numerical methods to find the dominant eigenpair of the matrix in ex.(1)

\begin{tabular}{|l|l|l|l|l|}
\hline \multirow{2}{*}{$\mathrm{k}$} & \multicolumn{1}{|c|}{ Approximate values of the dominant eigenvalue by the methods } \\
\cline { 2 - 5 } & \multicolumn{1}{|c|}{ Power } & \multicolumn{1}{|c|}{ Wilkinson } & \multicolumn{1}{|c|}{ Aitken } & Proposed \\
\hline 1 & 11 & 11 & & \\
3 & 7.7273 & 8.8655 & 8.9695 & 8.8655 \\
4 & 8.7294 & 8.8655 & 8.9108 & \\
5 & 9.2316 & & 8.8853 & \\
6 & 8.6329 & & 8.8742 & \\
7 & 9.0235 & & 8.8693 & \\
8 & 8.7627 & & 8.8671 & \\
9 & 8.9342 & & 8.8662 & \\
10 & 8.8203 & & 8.8658 & \\
11 & 8.8955 & & 8.8656 & \\
12 & 8.8457 & & 8.8655 & \\
13 & 8.8786 & & 8.8655 & \\
14 & 8.8568 & & & \\
15 & 8.8712 & & & \\
16 & 8.8617 & & & \\
17 & 8.8680 & & & \\
18 & 8.8638 & & & \\
19 & 8.8666 & & & \\
20 & 8.8647 & & & \\
21 & 8.8659 & & & \\
22 & 8.8651 & & & \\
23 & 8.8657 & & & \\
24 & 8.8653 & & & \\
25 & 8.8656 & & & \\
26 & 8.8654 & & & \\
27 & 8.8655 & & & \\
\hline R.T. & $00: 00: 015$ & $00: 00: 01$ & & \\
\hline Eigenvector & & & \\
\hline
\end{tabular}

Example (2):[2 ] consider the matrix

$$
A=\left[\begin{array}{rrr}
4 & -1 & 1 \\
-1 & 3 & -2 \\
1 & -2 & 3
\end{array}\right]
$$

The exact eigenvalues of $\mathrm{A}$ are $\lambda_{1}=6, \lambda_{2}=3, \lambda_{3}=1$ 
we compute $q=\frac{\lambda_{2}+\lambda_{3}}{2}=\frac{3+1}{2}=2$

The numerical values of this solution are presented in table (2).

Table (2): Results of the numerical methods to find the dominant eigenpair of the matrix in ex.(2)

\begin{tabular}{|c|c|c|c|c|}
\hline \multirow{2}{*}{$\mathrm{k}$} & \multicolumn{4}{|c|}{ Approximate values of the dominant eigenvalue by the methods } \\
\hline & Power & Wilkinson & Aitken & Proposed \\
\hline 1 & 4 & 4 & & \\
\hline 2 & 4.5000 & 5 & & \\
\hline 3 & 5 & 5.6667 & -Inf & 7.0003 \\
\hline 4 & 5.4000 & 5.9091 & 7.0000 & 6.0476 \\
\hline 5 & 5.6667 & 5.9767 & 6.2003 & 6.0028 \\
\hline 6 & 5.8235 & 5.9942 & 6.0472 & 6.0003 \\
\hline 7 & 5.9091 & 5.9985 & 6.0120 & 5.9999 \\
\hline 8 & 5.9538 & 5.9996 & 6.0027 & 6.0000 \\
\hline 9 & 5.9767 & 5.9999 & 6.0008 & 6.0000 \\
\hline 10 & 5.9883 & 6.0000 & 6.0002 & \\
\hline 11 & 5.9942 & 6.0000 & 6.0003 & \\
\hline 12 & 5.9971 & & 5.9999 & \\
\hline 13 & 5.9985 & & 5.9998 & \\
\hline 14 & 5.9993 & & 6.0004 & \\
\hline 15 & 5.9996 & & 5.9998 & \\
\hline 16 & 5.9998 & & 6.0002 & \\
\hline 17 & 5.9999 & & 6.0000 & \\
\hline 18 & 6.0000 & & 5.9997 & \\
\hline 19 & 6.0000 & & 6.0000 & \\
\hline R.T. & 00:00:016 & 00:00:016 & 00:00:016 & 00:00:015 \\
\hline \multicolumn{2}{|c|}{ Eigenvector } & \multicolumn{3}{|c|}{$\begin{array}{lll}1.0000 & -1.0000 & 1.0000\end{array}$} \\
\hline
\end{tabular}

Example (3):[7] Let

$$
A=\left[\begin{array}{lll}
2 & 3 & 1 \\
5 & 4 & 8 \\
0 & 8 & 1
\end{array}\right]
$$

The exact eigenvalues of $\mathrm{A}$ are $\lambda_{1}=11.8194, \lambda_{2}=1.3111, \lambda_{3}=-6.1305$ we compute $q=\frac{1.3111-6.1305}{2}=-2.4097$

The numerical values of this solution are presented in table (3). 
Table (3): Results of the numerical methods to find the dominant eigenpair of the matrix in ex.(3)

\begin{tabular}{|l|l|l|l|l|}
\hline \multirow{2}{*}{$\mathrm{k}$} & \multicolumn{4}{|c|}{ Approximate values of the dominant eigenvalue by the methods } \\
\cline { 2 - 5 } & Power & \multicolumn{1}{|c|}{ Wilkinson } & Aitken & Proposed \\
\hline 1 & 17 & 17 & & \\
2 & 10 & 10.8690 & 12.0710 & 11.8926 \\
3 & 12.9412 & 12.0977 & 11.8831 & 11.8274 \\
4 & 11.2886 & 11.7511 & 11.8363 & 11.8206 \\
5 & 12.1077 & 11.8381 & 11.8239 & 11.8197 \\
6 & 11.6734 & 11.8147 & 11.8206 & 11.8195 \\
7 & 11.8961 & 11.8207 & 11.8197 & 11.8194 \\
8 & 11.7799 & 11.8191 & 11.8195 & 11.8194 \\
9 & 11.8400 & 11.8195 & 11.8194 & \\
10 & 11.8088 & 11.8194 & & \\
11 & 11.8249 & 11.8194 & & \\
12 & 11.8165 & & & \\
13 & 11.8209 & & & \\
14 & 11.8186 & & & \\
15 & 11.8198 & & & \\
16 & 11.8192 & & & \\
17 & 11.8195 & & & \\
18 & 11.8193 & & & \\
19 & 11.8194 & & & \\
20 & 11.8194 & & & \\
\hline R.T. & $00: 00: 015$ & $00: 00: 016$ & & \\
\hline Eigenvector & 0.3808 & $1.0000: 015$ & \\
\hline
\end{tabular}

Example (4):[10 ] Let

$A=\left[\begin{array}{rrr}1 & 4 & 6 \\ -3 & 4 & 3 \\ 2 & -1 & 5\end{array}\right]$

The exact eigenvalues of $\mathrm{A}$ are

$\lambda_{1}=7, \lambda_{2}=1.5000+2.9580 \mathrm{i}, \lambda_{3}=1.5000-2.9580 i$

and $q=1.5$ 
The numerical values of this solution are presented in table (4).

Table (4): Results of the numerical methods to find the dominant eigenpair of the matrix in ex.(4)

\begin{tabular}{|l|l|l|l|l|}
\hline \multirow{2}{*}{$\mathrm{k}$} & \multicolumn{4}{|c|}{ Approximate values of the dominant eigenvalue by the methods } \\
\cline { 2 - 5 } & Power & Wilkinson & Aitken & Proposed \\
\hline 1 & 11 & 11 & & \\
2 & 5.7273 & 4.8947 & 5.7929 & 6.6054 \\
3 & 5.7937 & 7.2713 & 5.7236 & 6.7771 \\
4 & 7.0575 & 6.6474 & 6.9179 & 6.9710 \\
5 & 6.9006 & 7.3197 & 6.9552 & 7.0043 \\
6 & 6.9843 & 6.7254 & 7.0391 & 6.9237 \\
7 & 7.0174 & 7.0230 & 6.9977 & 6.9772 \\
8 & 6.9690 & 6.9688 & 6.9832 & 6.9968 \\
9 & 6.9891 & 7.0266 & 7.0111 & 6.9996 \\
10 & 6.9996 & 6.9759 & 6.9987 & 6.9931 \\
11 & 6.9986 & 7.0019 & 6.9992 & 6.9981 \\
12 & 7.0004 & 6.9974 & 7.0000 & 6.9997 \\
13 & 6.9999 & 7.0022 & & 7.0000 \\
14 & 6.9996 & 6.9980 & 7.0002 & \\
15 & 7.0000 & 6.9998 & & \\
16 & & 7.0000 & & \\
17 & \multicolumn{5}{|c|}{$00: 00: 016$} & \\
\hline R.T. & \multicolumn{5}{|l|}{0.0000} & 1.0000 & \\
\hline Eigenvector & 1.0000 & & \\
\hline
\end{tabular}

Example (5):[6] consider the matrix

$$
\mathrm{A}=\left[\begin{array}{rrrr}
2.5 & -2.0 & 2.5 & 0.5 \\
0.5 & 5.0 & -2.5 & -0.5 \\
-1.5 & 1.0 & 3.5 & -2.5 \\
2.0 & 3.0 & -5.0 & 3.0
\end{array}\right]
$$

The exact eigenvalues of $\mathrm{A}$ are $\lambda_{1}=6, \lambda_{2}=4, \lambda_{3}=3, \lambda_{4}=1$

we compute $q=\frac{\lambda_{2}+\lambda_{4}}{2}=2.5$

The numerical values of this solution are presented in table (5). 
Table (5): Results of the numerical methods to find the dominant eigenpair of the matrix in ex.(5)

\begin{tabular}{|l|l|l|l|l|}
\hline \multirow{2}{*}{$\mathrm{k}$} & \multicolumn{3}{|c|}{ Approximate values of the dominant eigenvalue by the methods } \\
\cline { 2 - 5 } & \multicolumn{1}{|c|}{ Power } & \multicolumn{1}{|c|}{ Wilkinson } & Aitken & Proposed \\
\hline 1 & 3.5000 & 4.5000 & & \\
2 & 6.0000 & 8.6250 & 8.6470 & 6.8306 \\
3 & 7.2857 & 5.4490 & 6.9352 & 6.3226 \\
4 & 6.8039 & 6.6540 & 5.8780 & 6.1898 \\
5 & 6.4870 & 5.8988 & 6.0454 & 6.0603 \\
6 & 6.3025 & 6.1042 & 6.0295 & 6.0273 \\
7 & 6.1924 & 5.9814 & 6.0139 & 6.0100 \\
8 & 6.1243 & 6.0187 & 6.0069 & 6.0048 \\
9 & 6.0812 & 5.9966 & 6.0029 & 6.0018 \\
10 & 6.0534 & 6.0034 & 6.0015 & 6.0009 \\
11 & 6.0353 & 5.9994 & 6.0006 & 6.0003 \\
12 & 6.0234 & 6.0006 & 5.9999 & 6.0002 \\
13 & 6.0155 & 5.9999 & 6.0003 & 6.0001 \\
14 & 6.0103 & 6.0001 & 6.0005 & 6.0000 \\
15 & 6.0069 & 6.0000 & 5.9998 & 6.0000 \\
16 & 6.0046 & 6.0000 & 5.9993 & \\
17 & 6.0030 & & 6.0003 & \\
18 & 6.0020 & & 6.0005 & \\
19 & 6.0014 & & 5.9984 & \\
20 & 6.0009 & & 6.0002 & \\
21 & 6.0006 & & 6.0000 & \\
22 & 6.0004 & & 6.0002 & \\
23 & 6.0003 & & 6.0003 & \\
24 & 6.0002 & & 6.0001 & \\
25 & 6.0001 & & 6.0000 & \\
26 & 6.0001 & & & \\
27 & 6.0000 & & & \\
28 & 6.0000 & & & \\
\hline R.T. & $00000: 016$ & $00: 00: 016$ & & \\
\hline Eigenvector & -0.5000 & 0.5000 & \\
\hline
\end{tabular}

\section{Discusions and Concluions}

The convergence of Power method of first degree and the speed of convergence is slow so that more iteration steps are needed, it is the simplest iterative method. The Aitken and Wilkinson methods converge faster than the Power method. The Wilkinson method requires that all the eigenvalues are real and the Aitken method requires more computations 
per iteration compard with the Power method and Wilkinson method. The hybrid algorithm converges faster than the Power, Wilkinson and Aitken methods and requires more computations per iteration. It is quit insensitive to the properities of the matrices used and it evalutes the greatest eigenpair of any matrix.

All the algorithms are used the initial unit vector and the tolerance is $10^{-4}$, our numerical results, in tables $(1,2,3,4$ and 5$)$, indicate that the proposed algorithm performs better than the Power method and the results are given in Table (6). Specifically quoting the number of iteration (NOI) and running computer time (R.T.) as a basis of the comparison.; namely taking the Power method as $100 \%$ NOI the proposed method needs 35.8\% NOI while Aitken method needs 64.2\% NOI and Wilkinson method needs $50.9 \%$ NOI. Note that a clear looking to test example no. (4) it is obvious that the new method is stable for finding the dominant eigenvalue while the other methods are alternating to reach the solution with the same running time, and the Wilkinson method is very slow because some of eigenvalues of this example are complex.

Finally, we have noticed that the proposed method seems to be accurate, fast, and gives very encouraging numerical results for the considered matrices.

Table (6): Comparison between the suggested method and the Power, Wilkinson and Aitken methods.

\begin{tabular}{|l|l|l|l|l|}
\hline $\begin{array}{c}\text { No. of } \\
\text { examples }\end{array}$ & $\begin{array}{c}\text { NOI of } \\
\text { Power }\end{array}$ & $\begin{array}{c}\text { NOI of } \\
\text { Wilkinson }\end{array}$ & $\begin{array}{c}\text { NOI of } \\
\text { Aitken }\end{array}$ & $\begin{array}{c}\text { NOI of } \\
\text { Proposed }\end{array}$ \\
\hline 1 & 27 & 2 & 10 & 1 \\
\hline 2 & 18 & 10 & 15 & 6 \\
\hline 3 & 19 & 10 & 8 & 6 \\
\hline 4 & 15 & 17 & 11 & 12 \\
\hline 5 & 27 & 15 & 24 & 13 \\
\hline Total & 106 & 54 & 68 & 38 \\
\hline
\end{tabular}

(a)

\begin{tabular}{|l|l|l|l|}
\hline $\begin{array}{c}\text { Power } \\
\text { method }\end{array}$ & $\begin{array}{c}\text { Wilkinson } \\
\text { method }\end{array}$ & $\begin{array}{c}\text { Aitken } \\
\text { method }\end{array}$ & $\begin{array}{c}\text { Proposed } \\
\text { method }\end{array}$ \\
\hline $100 \%$ & $50.9 \%$ & $64.2 \%$ & $35.8 \%$ \\
\hline
\end{tabular}

(b) 


\section{References}

1) AL-Bayati, A. Y. and Ahmed, E. S. (2005), "A New Numerical Procedure for Solving Linear System". Tikrit Journal of Pure Science, Vol. (lo), No. (1), pp.173-176.

2) Burden, R. L. and faires, J. D. (1993), "Introduction to Numerical Analysis", PWS Publishing Company, U.S.A.

3) Canright, Geoffrey, Kenth Engo-Monsen and Mark J. (2005), "Efficient and Robust Fully Distribnted Power Method with an Application to Link Aralysis", Technical Report UBLCS http: // www.cs .unibo.it /.

4) Descloux, Jean and Jacques Rappaz (1983), "A Nonlinear Inverse Power Method with Shift", SIAM Journal on Numerical Analysis, Vol. 20, No. 6, pp. 1147-1152.

5) Jennings, Alan (1971), "Accelerating the Convergence of Matrix Iterative Processes" IMA Journal of Applied Mathematics, 8(1), pp. 99-110.

6) Mathews, J. H. and fink, K.D (1999), "Numerical Methods Using Matlab", prentice-Hall, Inc.

7) Press, William H., Saul A. T., William T. V. and Brian P. Flannery (2006) "Numerical Recipes in Fortran 77", Volume (1), part (2), Cambridge University Press.

8) Reich, S. (1970), "On Aitkin's $\Delta_{2}$-Method", Amer. Math. Monthly 77, pp. 203-304

9) Riess, R. D. (1971), "Introduction of a Conditioning Parameter in Computing Characteristic Equations", Proceedings of the IEEE, Volume: 59, No.8, pp.: 1275- 1276.

10) Shanthakumar, M. (1989), "Computer Based Numerical Analysis" Khanna Publishers. INDIA

11) Sun, Huan and Yinin Wei (2006), "A Note on the PageRank Algorithm", Applied Mathematics and Computation 179, pp. 799806.

12) Wilkinson, J. H. (1958), "The Calculation of Eigenvectors by the Method of Lanczos", National Physical Laboratory, Teddington, UK, 1(3), pp. 148-152.

13) Wood, D. M. and A. Zunger (1985), "A New Method for Diagonalising Large Matrices", J. Phys. A: Math. Gen. 18(9), pp. 1343-1359. 